\title{
A Dual Gender Rare Case with 47,XY, + 18/46,XX Karyotype: Chimera or Mosaic?
}

\author{
Ravindran Ankathil ${ }^{1}$ Foong Eva ${ }^{1}$ Zulaikha Abu Bakar ${ }^{1} \quad$ Nazihah Mohd Yunus $^{1} \quad$ Nurul Alia Nawi ${ }^{1}$ \\ Aziati Annuar ${ }^{1}$ Cheng Yi Ting $^{1}$ Sarina Sulong ${ }^{1}$ \\ ${ }^{1}$ Human Genome Centre, School of Medical Sciences, Universiti Sains \\ Malaysia, Kubang Kerian, Kelantan, Malaysia \\ J Child Sci 2021;11:e41-e44. \\ Address for correspondence Ravindran Ankathil, PhD, Human \\ Genome Centre, School of Medical Sciences, Universiti Sains Malaysia, \\ 16150, Kubang Kerian, Kelantan, Malaysia \\ (e-mail: rankathil@hotmail.com).
}

\begin{abstract}
Keywords

- chimerism

- dual gender

- liveborn

- trisomy 18

Our objective is to report one rare case of dual gender chimerism involving abnormal male trisomy 18 and normal female karyotype. The baby was born full term with birth weight of $1.8 \mathrm{~kg}$, not vigorous with light meconium stained liquor and Apgar score of $5^{1}, 8^{5}$ and $9^{10}$. Parents are 40 years old and mother is $\mathrm{G} 6 \mathrm{P} 5+1$. The baby had clinical features of Edwards syndrome, and a blood sample was sent to Human Genome Centre, Universiti Sains Malaysia, Malaysia for cytogenetic analysis. Conventional cytogenetic analysis results showed two distinct sex discordant genetic cell lines $X Y$ and $X X$ in 90:10 ratio. The male genetic cell line $X Y$ also showed trisomy $18(47, X Y,+18)$ consistent with clinical diagnosis of male Edwards syndrome, whereas the second genetic cell line showed normal 46,XX female. The present case was reported as dual gender chimera with chi 47,XY, +18/46,XX karyotype pattern. To the best of available knowledge, dual gender chimerism with abnormal male trisomy 18 and normal female karyotype has not been reported so far, and this case is reported for its rarity and as the first report.
\end{abstract}

\section{Introduction}

Individuals who have two or more distinct populations of cells in the body can either be labeled as a mosaic or chimera depending on the mechanism of formation of these different cell lines. Mosaicism is referred to when the different cell lines arise from the same zygote, usually resulting from inaccurate segregation during mitosis. On the other hand, the different cell lines in chimera originate from different zygotes in which two zygotes fuse into one during an early embryonic stage producing a mixture of completely unrelated two cell lineages. ${ }^{1}$ Human chimerism is a rare genetic condition. It is expected that many chimeras are in fact healthy and are almost never detected, especially if both zygotes are of the same genetic sex unless they exhibit abnormalities such as mismatched eyes color, partly- colored hair, uneven skin pigmentation, or hermaphroditic genitalia. Otherwise, many are incidentally identified by blood group or paternity/maternity testing or other clinical investigations related to health issues. Chimerism is often recognized cytogenetically when they are of dual gender. Dual gender chimeras (46,XX/46,XY cell lines) account for $13 \%$ of true hermaphrodites and are diagnosed at birth due to ambiguous external genitalia. ${ }^{2}$ Rarely dual gender chimerism has also been reported in cases in which a normal cell lineage (disomy) coexists with an abnormal (trisomy) one, each causing a distinct sex chromosome complement. Few rare disomy/trisomy chimeras with abnormal trisomy 21 and normal disomy karyotypes were reported. ${ }^{2}$ Here we report an extremely rare case of dual gender trisomy/disomy chimerism with abnormal male trisomy 18 and normal female karyotype. received

September 20, 2020

accepted after revision

November 27, 2020
(C) 2021. The Author(s).

This is an open access article published by

Thieme under the terms of the Creative

Commons Attribution License, permitting

unrestricted use, distribution, and

reproduction so long as the original work is

properly cited. (https://creativecommons.org/

licenses/by/4.0/)

Georg Thieme Verlag KG, Rüdigerstraße

14, 70469 Stuttgart, Germany
DOI https://doi.org/ 10.1055/s-0040-1722275. ISSN 2474-5871. 


\section{Case History}

A full-term baby boy was born to a 40 -year-old woman who was gravida 6 para 5 . She had a history of non-identical male twin pregnancy in the year 2000 and one abortion in the year 2014. The proband was born via spontaneous vaginal delivery, and the labor was uneventful. He was born not vigorous with Apgar score of 5 at 1 minute, 8 at 5 minutes, and 9 at 10 minutes $\left(5^{1}, 8^{5}\right.$ and $\left.9^{10}\right)$; he required resuscitation. He had low birth weight of $1.87 \mathrm{~kg}$ and appeared dysmorphic with wide anterior fontanelle, flat occiput, triangular facies, hypertelorism, depressed nasal bridge, micrognathia, underdeveloped left ear, low set ears, wide spaced nipple, trisomy hands, bilaterally flexed wrist, and bilateral rocker bottom feet. Examination of the genitalia revealed small prepuce, left descended testes, and right undescended testes. Right testis was palpable at right inguinal region. He was clinically diagnosed to have Edwards syndrome, and his peripheral blood sample was sent for cytogenetic analysis. Family counseling was performed and the child was managed conservatively, but he died 6 days postbirth.

\section{Conventional Cytogenetic Analysis and Results}

G-Banding Using Trypsin and Giemsa (GTG)-banded chromosomal analysis performed on 80 GTG banded metaphases revealed chi 47,XY,+ 18 [72]/46,XX [8] (-Figs. 1 and 2) karyotype pattern with dual gender cell lines $\mathrm{XY}$ and $\mathrm{XX}$ in 90:10 ratio. The cytogenetic result was consistent with dual gender chimerism involving abnormal male trisomy 18 (Edwards syndrome) karyotype and a normal female 46,XX karyotype pattern.

\section{Discussion}

Dual gender chimeras are very rare, as symptomatic chimera with high proportion of trisomic chimeric cells in the body are unlikely to survive. Only a few chimera cases of trisomy 21 and normal karyotype have been reported. ${ }^{2-4}$ To the best of our knowledge, dual gender chimerism with abnormal male trisomy 18 coexisting with normal female karyotype has not been reported so far, and this case is reported for its rarity and as the first report. Trisomy 18 is the second most common autosomal trisomy syndrome after trisomy 21 and accounts for high frequency of fetal loss and infant mortality. A possible explanation on the rarity of dual gender chimerism with trisomy 18 and normal karyotype may be that the chimera is asymptomatic due to the low proportion of trisomic chimeric cells in the body and due to the trisomic chimeric cells being confined to only certain organs. Another possibility is that the chimera is unable to survive due to the high proportion of trisomic chimeric cells in the body involving nearly every organ system in the body.

Sex-discordant XX/XY chimeras can have variable genital phenotypes, ranging from a normal male or female phenotype to different degrees of ambiguous genitalia ${ }^{1}$ based on the percentage and location of the abnormal cells present in the body. In a study by Madan $^{5}$ on 50 individuals with a 46,XX/46,XY karyotype, only 28 were either true hermaphrodites or had

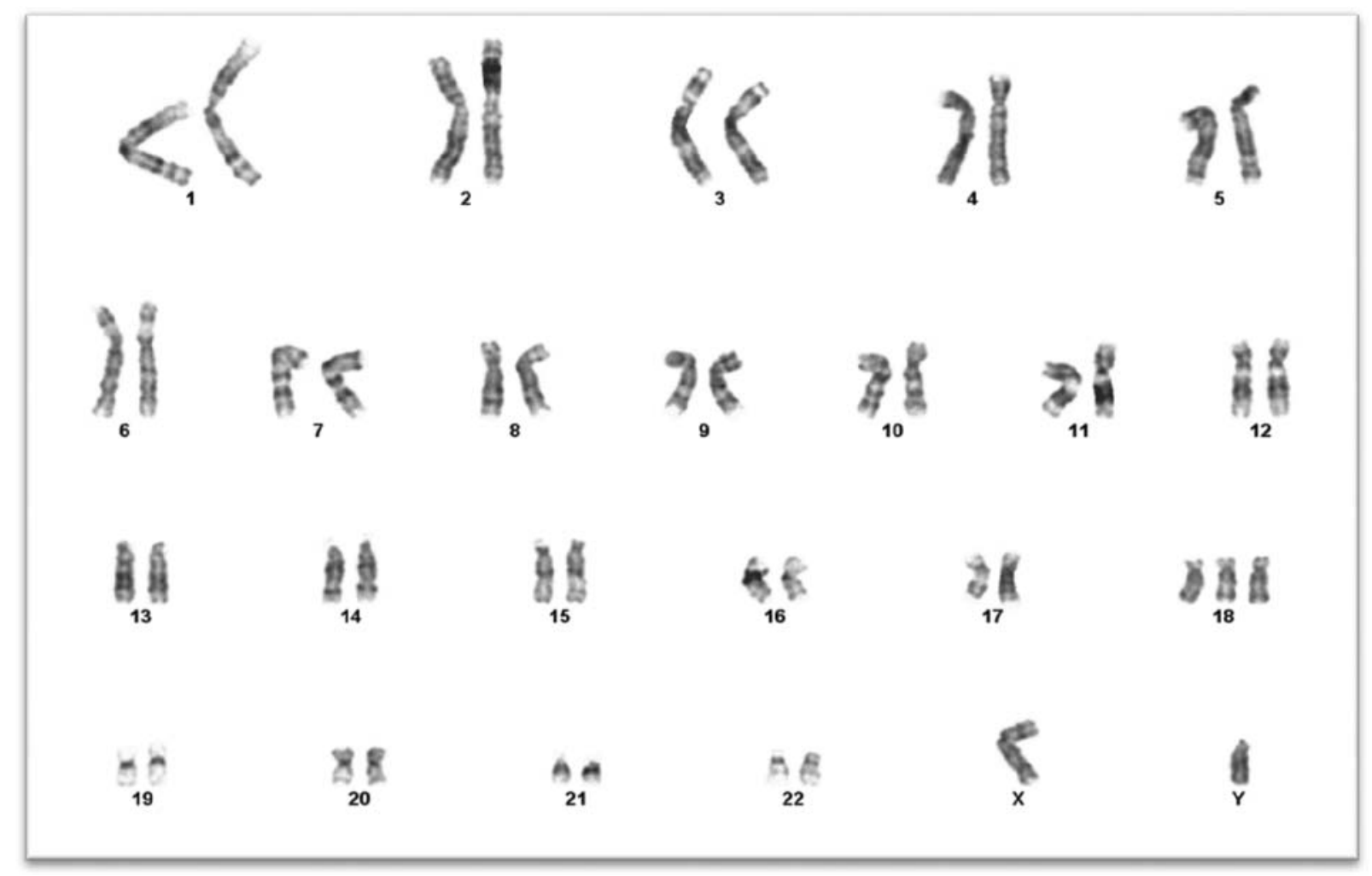

Fig. 1 Karyotype of the newborn dual gender chimeric child showing 47,XY, + 18 karyotype pattern. 


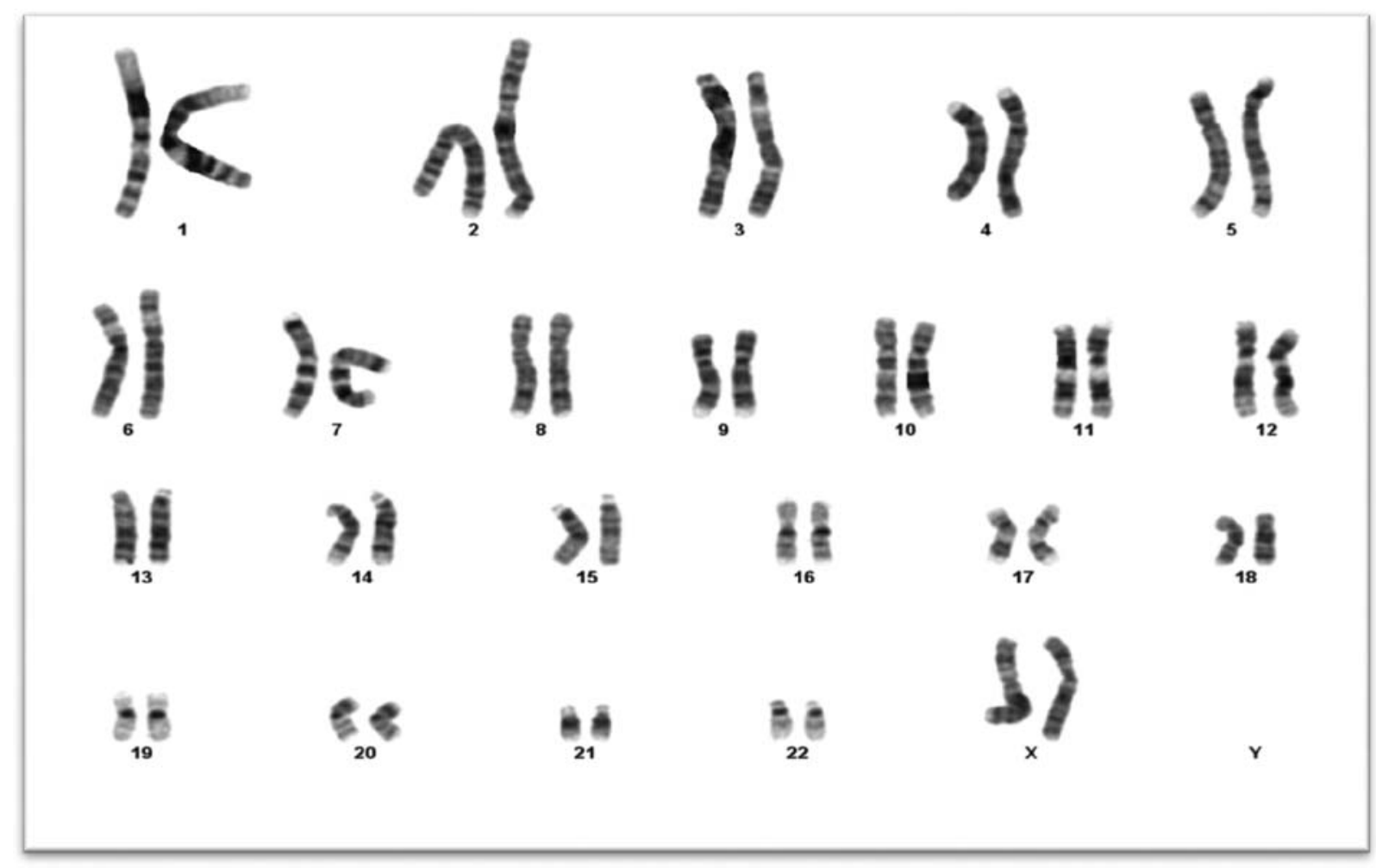

Fig. 2 Karyotype of the newborn dual gender chimeric child showing normal 46,XX female karyotype pattern.

ambiguous genitalia. Van Bever et $\mathrm{al}^{6}$ also reported a phenotypically male patient presented at the age of 19 with a painless right scrotal mass and subsequently diagnosed with 46,XX/46, $\mathrm{XY}$ ovotesticular-disorders of sex development (OT-DSD). Overall, 10 to $33 \%$ of patients with OT-DSD have the $46, \mathrm{XX} / 46, \mathrm{XY}$ karyotype pattern. ${ }^{7}$ The present case is a phenotypically male baby whose peripheral blood CCA showed dual gender chimerism involving 90\% abnormal male trisomy 18 (Edwards syndrome) karyotype and 10\% normal female 46, XX karyotype pattern.

Human chimeras have been broadly divided into two groups, namely naturally acquired chimerism and artificial chimerism. Naturally acquired chimerism takes place in utero, whereas artificial chimerism is acquired following blood transfusion or following organ, stem cell, and bone marrow transplantation. Naturally acquired microchimerism commonly occurs during pregnancy when the placenta is imperfect, and there is reciprocal exchange of cells between a mother and her child. This often leads to the stable engraftment of hematopoietic and nonhematopoietic stem cells in both parties ${ }^{8}$ resulting in fetal-maternal chimera.

Examples of naturally acquired macrochimerism are blood chimera, tetragametic chimera, parthenogenetic chimeras, gynogenetic chimera, and androgenetic chimeras. Blood chimera, also known as twin chimera, occurs when there is blood anastomoses formed between the placentas of dizygotic twins, allowing exchange of cells and genetic material between the fraternal twins via in utero. The transfer of stem cells between the developing embryos lead to one or both of the embryos containing two genetically distinct cell populations. Subsequent to the first identification of human blood chimera in $1953,{ }^{9}$ more human blood chimera has been identified. ${ }^{10-12}$

Meanwhile, postzygotic fusion of the two different embryos can give rise to tetragametic or dispermic chimerism. This condition arises from four gametes: two eggs fertilized by two sperms, resulting in dizygotic twins who fuse into one individual. When a woman is pregnant with fraternal twins and one of the embryo dies very early on, the other surviving embryo can "absorb" its twin's cells, a process known as vanishing twin syndrome. The fetus who survives will end up with two sets of DNA. As the fetus develops, he/she possess organs that have different sets of chromosomes. In addition, postzygotic fusion of a fertilized ovum with a fertilized second polar body also can produce tetragametic chimeras. Parthenogenetic (trigametic) chimeras, gynogenetic chimeras, and androgenetic chimeras also are resulted from endoreplication of one of the gametic genomes. ${ }^{5}$

Sex chromosome discordance is also observed in patients with mosaicism. In mosaicism, two or more separate cell lines originate from single zygote. It is a consequence of errors in segregation which either resulted from nondisjunction or anaphase lag. In the present case, the possibility of nondisjunction resulting in $48, \mathrm{XXY},+18$ zygote followed by somatic loss of an $X$ chromosome and separately, loss of an $Y$ chromosome and chromosome 18 which resulted in the 47 , $\mathrm{XY},+18 / 46$, XX mosaic karyotype cannot be ruled out. Unfortunately, further molecular investigations could not be initiated as the baby passed away. Had DNA been available, molecular analysis such as SNP array analysis and 
microsatellite marker analysis could have been performed to investigate the parental origin of this patient's genome. With this, the dual gender cell lines which result either from chimerism or from mosaicism could have been determined. Once chimerism was confirmed, the type of chimerism also could have been determined. In view of no molecular analysis available to support possibility of mosaicism, we concluded this patient as dual gender tetragametic chimera.

We propose two postulations on the origin of this dual gender chimera. One strong possibility is that the mother had an undiagnosed fraternal twin pregnancy, and the female embryo died in utero in very early stage of pregnancy. Some of the cells of this female embryo might have been absorbed by the surviving twin. The second possibility is that this could be a blood chimera as the diagnosis was made by conventional cytogenetic analysis where cells from leucocytes were used for examination. The possibility is that only exchange of hematopoietic stem cells had occurred between the twins in utero before the female embryo died in utero. If that was the case, only the hematopoietic cell lines might have had dual gender sets of different DNA. The only method to differentiate both the condition is by examining other tissues in the body for the presence of different sets of DNA. Unfortunately, this could not be done as this newborn passed away after a few days of life and before the completion of cytogenetic analysis.

Research suggests that the incidence of vanishing twin syndrome occurs before the 12th week of pregnancy in around $36 \%$ of pregnancies with two gestations and more than $50 \%$ of pregnancies with three or more gestations. ${ }^{13-17}$ The incidence of chimerism further increases due to assisted fertility procedures involving artificially induced ovulation. This leads to increased frequency of twinning in all pregnancies. In in vitro fertilization, some feto-maternal specialists tend to transfer more than one embryo into the uterus to increase the chance of successful implantation. Thus, the frequency of twinning increases.

In this chimera, the surviving fetus happened to be the abnormal male trisomy18 and not the normal female. As chromosomal aneuploidies are the most common etiology for pregnancy loss, the male twin with trisomy 18 was expected to undergo spontaneous abortion, and the normal female twin was expected to survive. It is presumed that this female twin might have had a lethal de novo mutation which lead to death in early pregnancy.

The parents have been counseled on the nature, consequences, and general management of children with Edwards syndrome. However, the chimeric child passed away a few days after birth. The parents were also informed on the probability and risk of occurrence of other aneuploidy in future pregnancies in view of advanced maternal age. Information and importance of fetal aneuploidy testing to screen for common aneuploidies in future pregnancies was offered to this couple.

\section{Funding}

None.

\section{Conflict of Interest}

None declared.

\section{Acknowledgments}

The authors would like to thank all staff of Cytogenetic Laboratory of the Human Genome Centre, School of Medical Sciences, Universiti Sains Malaysia for their technical assistance in chromosome analysis.

\section{References}

1 Kawamura R, Kato T, Miyai S, et al. A case of a parthenogenetic 46, $\mathrm{XX} / 46, \mathrm{XY}$ chimera presenting ambiguous genitalia. J Hum Genet 2020;65(08):705-709

2 Lee KF, Hsu CS, Kuo PL, Chen JL, Jiang YH, Liu IY. The identification of a spontaneous $47, \mathrm{XX},+21 / 46, \mathrm{XY}$ chimeric fetus with male genitalia. BMC Med Genet 2012;13(01):85

3 Sawai T, Yoshimoto M, Kinoshita EI, et al. Case of 46, XX/47, XY,+ 21 chimerism in a newborn infant with ambiguous genitalia. American journal of medical genetics 1994;49(04):428-430

4 Lucon DR, Zanchetta LM, Cavalcanti DP. Chimerism 47, XY,+ $21 / 46, X X$ in a female infant with anencephaly and other congenital defects. Genetics and molecular biology 2006;29(01):36-37

5 Madan K. Natural human chimeras: A review. Eur J Med Genet 2020;63(09):103971

6 van Bever Y, Wolffenbuttel KP, Bruggenwirth HT, et al. Multiparameter investigation of a $46, \mathrm{XX} / 46, \mathrm{XY}$ tetragametic chimeric phenotypical male patient with bilateral scrotal ovotestes and ovulatory activity. Sex Dev 2018;12(1-3)145-154

7 Winberg J, Gustavsson P, Lagerstedt-Robinson K, et al. Chimerism resulting from parthenogenetic activation and dispermic fertilization. Am J Med Genet A 2010;152A(09):2277-2286

8 Eikmans M, van Halteren AG, van Besien K, van Rood JJ, Claas FH. Naturally acquired microchimerism: implications for transplantation outcome and novel methodologies for detection. Chimerism 2014;5(02):24-39

9 Dunsford I, Bowley C, Hutchison AM, Thompson JS, Sanger R, Race R. Human blood-group chimera. BMJl 1953;2(4827):81

10 Bird G, Battey D, Greenwell P, Mortimer C, Watkins W, Wingham J. Further observations on the Birmingham chimaera. BMJ 1976;13 (01):70-71

11 Watkins WM, Yates AD, Greenwell P, et al. A human dispermic chimaera first suspected from analyses of the blood group genespecified glycosyltransferases. J Immunogenet 1981;8(02): $113-128$

12 Wolinsky H. A mythical beast. Increased attention highlights the hidden wonders of chimeras. EMBO Rep 2007;8(03):212-214

13 Yu N, Kruskall MS, Yunis JJ, et al. Disputed maternity leading to identification of tetragametic chimerism. N Engl J Med 2002;346 (20):1545-1552

14 Danielsson K. Vanishing Twin Syndrome Overview. Treasure Island, FL: StatPearls Publishing; 2018

15 Davies MJ, Rumbold AR, Whitrow MJ, et al. Spontaneous loss of a co-twin and the risk of birth defects after assisted conception. J Dev Orig Health Dis 2016;7(06):678-684

16 Márton V, Zádori J, Kozinszky Z, Keresztúri A. Prevalences and pregnancy outcome of vanishing twin pregnancies achieved by in vitro fertilization versus natural conception. Fertil Steril 2016; 106(06):1399-1406

17 Zhou L, Gao X, Wu Y, Zhang Z. Analysis of pregnancy outcomes for survivors of the vanishing twin syndrome after in vitro fertilization and embryo transfer. Eur J Obstet Gynecol Reprod Biol 2016; 203:35-39 